\title{
Estudio clínico de la hepatitis autoinmune del adulto en Valencia
}

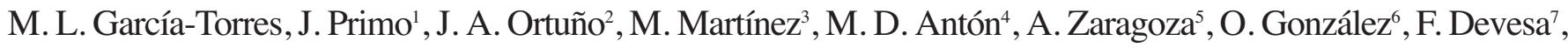 \\ C. Merino y J. A. del Olmo
}

Servicio de Hepatología. Hospital Clínico Universitario. Departamento de Medicina. Valencia. ${ }^{2}$ Unidad de Digestivo. Servicio de Medicina Interna. Hospital de Sagunto. ${ }^{2}$ Servicio de Medicina Digestiva. Hospital Universitario La Fe. ${ }^{3}$ Servicio de Aparato Digestivo. Hospital General Universitario. ${ }^{4}$ Servicio de Aparato Digestivo. Hospital Universitario Doctor Peset. ${ }^{5}$ Unidad de Digestivo. Servicio de Medicina Interna. Hospital Lluis Alcanyis. Xátiva. ${ }^{6}$ Servicio de Aparato Digestivo. Hospital Arnau de Vilanova. ${ }^{7}$ Unidad de Digestivo. Servicio de Medicina Interna. Hospital Francesc de Borja. Gandía, Valencia

\section{RESUMEN}

Introducción: existen factores geográficos que influyen en las características de la hepatitis autoinmune (HAI).

Objetivo: conocer las características epidemiológicas, clínicas y respuesta al tratamiento de la hepatitis autoinmune en la provincia de Valencia.

Material y métodos: se trata de un estudio realizado en ocho hospitales de Valencia, recogiendo de forma prospectiva los casos de hepatitis autoinmune diagnosticados en el año 2003 y de forma retrospectiva los diagnosticados entre 1994 y 2002.

Resultados: se incluyeron 81 pacientes con HAI, siendo el $94 \%$ mujeres. La forma de presentación fue: $43 \%$ asintomático, $27 \%$ hepatitis aguda y $30 \%$ enfermedad hepática crónica. La mayoría (90\%) fueron del tipo 1 , mientras que las de tipo 2 fueron más jóvenes ( $p=0,007)$ y con predominio de hepatitis aguda ( $p$ $=0,04)$. Existía otra enfermedad autoinmune en uno de cada 4 casos. Al momento del diagnóstico una tercera parte de los pacientes presentaba cirrosis. La cirrosis se presentó sobre todo en pacientes de más de 60 años $(p<0,01)$ y sin diferencias según la formas clínica $(p=0,1)$. El tratamiento se indicó en 57 casos y con respuesta -completa o parcial- en el $87,7 \%$. No se encontraron diferencias significativas en la respuesta terapéutica según la forma de presentación ni los tipos de hepatitis autoinmune.

Conclusiones: en nuestra zona la hepatitis autoinmune del adulto se presenta sobre todo en mujeres y de manera asintomática. La mayoría es tipo 1 y en el $25 \%$ de los casos coexiste otra enfermedad autoinmune. Al diagnóstico la tercera parte presenta cirrosis hepática, sobre todo en los que tienen más de 60 años.

Palabras clave: Diagnóstico de la hepatitis autoinmune. Terapéutica de la hepatitis autoinmune. Epidemiología. Cirrosis hepática.

\begin{abstract}
Objective: the clinical phenotype of autoimmune hepatitis $(\mathrm{AIH})$ varies among geographical areas. The aim of this study is to determine the salient features of $\mathrm{AIH}$ in adult patients from the province of Valencia, Spain.

Material and methods: eighty-one patients with $\mathrm{AlH}$ attended to in eight acute-care hospitals between 1994 and 2003. New patients diagnosed with AIH during year 2003 were evaluated prospectively. Data from patients currently attending follow-up visits and diagnosed before 2003 were collected retrospectively.

Results: a total of $94 \%$ of patients were females. Forty-three percent were asymptomatic, $27 \%$ had acute hepatitis, and $30 \%$ had chronic hepatitis. Type $1 \mathrm{AIH}$ was diagnosed in $90 \%$ of cases. Type $2 \mathrm{AIH}$ was more frequent in younger patients, and presented with an acute pattern. One third of patients had cirrhosis at onset. Patients with cirrhosis were older than 60 years more frequently. Immunosuppressants were given to 57 patients, with complete or partial remission in $87.7 \%$. There were no significant differences in response to immunosuppression according to presentation pattern or AIH subtype.

Conclusions: $\mathrm{AIH}$ in Valencia was predominantly diagnosed in asymptomatic women. Most cases were type 1 , and in $25 \%$ of patients another autoimmune disease coexisted. At the time of diagnosis one third of patients had cirrhosis, particularly those over 60 years.
\end{abstract}

Key words: Autoimmune hepatitis diagnosis. Autoimmune hepatitis pathology. Autoimmune hepatitis drug therapy. Epidemiology. Liver cirrhosis.

García-Torres ML, Primo J, Ortuño JA, Martínez M, Antón MD, Zaragoza A, González O, Devesa F, Merino C, del Olmo JA. Estudio clínico de la hepatitis autoinmune del adulto en Valencia. Rev Esp Enferm Dig 2008; 100: 400-404.

Recibido: 19-02-08.

Aceptado: 25-03-08.

Correspondencia: Juan A. del Olmo. Servicio de Hepatología. Hospital Clínico Universitario. Avda. Blasco Ibáñez, 17. 46410 Valencia. e-mail: juan.a.del-olmo@uv.es

\section{INTRODUCCIÓN}

La hepatitis autoinmune (HAI) es una enfermedad de causa desconocida, que ocurre en niños y adultos de todas las edades y que suele evolucionar de forma progresiva. El diagnóstico se realiza por las características clínicas y ha- 
llazgos bioquímicos, así como niveles de gammaglobulinas séricas, autoanticuerpos y anormalidades histológicas. La forma de presentación clínica es muy diversa. En unos casos existen alteraciones clínicas propias de la enfermedad hepática crónica, en otros casos se presentan como las hepatitis agudas, con ictericia y elevados valores de transaminasas, mientras que otros casos son clínicamente asintomáticos y sólo detectables por alteraciones analíticas hepáticas (1). Existen factores geográficos que influyen no sólo en las formas de presentación y frecuencia de la HAI, sino también en la evolución y respuesta al tratamiento (2-4). La HAI tipo I es la forma más común, mientras que la HAI tipo 2 es menos frecuente en los pacientes adultos, existiendo pocos estudios acerca de sus características clínicas, pronóstico y respuesta al tratamiento (5). Por otra parte, se producen con frecuencia recaídas tras la retirada de la medicación, lo cual obliga a realizar estudios acerca de la conveniencia de mantener la terapéutica durante largos periodos de tiempo (6).

Por ello hemos realizado un estudio, en el que han participado ocho hospitales de la provincia de Valencia, que tiene por objetivo conocer las características epidemiológicas, clínicas, formas de presentación, distribución de los tipos, lesiones histológicas, existencia de enfermedades asociadas y respuesta al tratamiento de la HAI en un periodo de 10 años.

\section{MATERIAL Y MÉTODOS}

El estudio ha sido promovido por el Grupo de Estudio de Enfermedades Hepáticas de la Sociedad Valenciana de Patología Digestiva y han participado los servicios de digestivo de atención a adultos de ocho hospitales. Se diseñó un protocolo de estudio para recoger los datos de todos los pacientes atendidos en los centros participantes, diagnosticados entre enero de 1994 y diciembre de 2003. El protocolo se comenzó a aplicar en enero de 2003, de forma que los nuevos pacientes diagnosticados de HAI durante ese año (18 casos) eran evaluados de forma prospectiva, mientras que los pacientes diagnosticados antes de 2003 (63 casos) se fueron recogiendo de forma retrospectiva durante las visitas de seguimiento.

Para el diagnóstico de HAI se siguieron los criterios del International Autoimmune Hepatitis Group (IAIHG), establecidos en 1993 (7) y revisados en 1999 (8), y se clasificaron los diagnósticos como probable (10-15 puntos pretratamiento o 12-17 puntos postratamiento) o definitivo ( $>$ 15 puntos pretratamiento o $>17$ puntos postratamiento). La HAI se dividió en tipo 1 ante la presencia de autoanticuerpos antinucleares (ANA) y/o antimúsculo liso (SMA) y tipo 2 si la positividad era a autoanticuerpos antimicrosomales hígado-riñón, LKM.1 $(9,10)$. No hemos realizado el diagnóstico de HAI tipo 3 al no disponer los años anteriores de determinaciones para antiantígeno soluble hepático (anti-SLA) ni anti-hígado-páncreas (anti-LP). Se excluyeron los casos con rasgos de autoinmunidad pero que pre- sentaran infección por virus B o C de la hepatitis, o sospecha de etiología metabólica (hemocromatosis hereditaria, enfermedad de Wilson o déficit de alfa-1-antitripsina).

El estudio histológico de las biopsias hepáticas se revisó atendiendo especialmente a las características definidas por la IAIHG $(7,8)$ (lesiones de la lámina limitante, infiltrado linfocitario, formación en rosetas de las células hepáticas, cambios biliares y otros cambios). También se evaluó el grado de actividad necroinflamatoria y fibrosis según los criterios de METAVIR Cooperative Study Group (11). Para la valoración de respuesta al tratamiento -remisión completa, parcial, sin respuesta o recaída- se siguieron las recomendaciones del IAIHG $(7,8)$.

Todos los protocolos de recogida fueron revisados por los comités de estudios clínicos hospitalarios y las biopsias hepáticas se practicaron tras información y consentimiento previo.

\section{Estudios estadísticos}

Los datos recogidos por el protocolo clínico (Tabla I) se centralizaron en una base de datos única para ser analizados por la misma persona utilizando un programa estadístico SPSS 12.0 (SPSS Inc. Headquarter, 233S Wacker Drive, $11^{\text {th }}$ floor, Chicago, Illinois 60606, EE.UU.) y se aplicó la Chi cuadrado $\left(\chi^{2}\right)$ (con corrección de Yate's

Tabla I. Parámetros recogidos en el protocolo

\begin{tabular}{l}
-Fecha de nacimiento \\
-Sexo \\
-Enfermedad autoinmune asociada \\
-Antecedentes familiares \\
-Forma de presentación clínica (hipertransaminasemia asintomática, hepatitis agu- \\
da, enfemedad hepática crónica: astenia, anorexia, ictericia, coluria, descompen- \\
sación de cirrosis hepática) \\
-Analítica sanguínea: \\
- Hemograma \\
- Química hemática: aminotransferasas, fosfatasas alcalinas, gammaglutamil- \\
$\quad$ transpeptidasa, bilirrubina, colesterol, triglicéridos, proteinograma, inmuno- \\
$\quad$ globulinas, hierro, índice de saturación de transferrina, ceruloplasmina, \\
- Alfa1antitripsina \\
- Autoanticuerpos: antinucleares, antimusculoliso, antimitocondriales, anti- \\
- Marcadores virales: VHB, VHC, VIH \\
- Tipo de hepatitis autoinmune \\
-Punción biopsia hepática: \\
- Fecha \\
- Lesiones: valor según el Grupo Internacional de Hepatitis Autoinmune \\
-Valor pretratamiento \\
-Diagnóstico pretratamiento \\
-Tratamiento \\
-Valor postratamiento \\
-Diagnóstico postratamiento \\
-Cambio diagnóstico \\
-Respuesta al tratamiento. Tiempo \\
-Recaída durante tratamiento de mantenimiento \\
-Recaída tras retirada de tratamiento \\
\hline
\end{tabular}


cuando era necesario), la probabilidad exacta de Fisher para variables cualitativas y el test t de Student y análisis de varianza (Friedman one-way ANOVA) para variables cuantitativas. Se consideraron significativos valores de $\mathrm{p}$ $<0,05$.

\section{RESULTADOS}

De 88 protocolos recogidos de pacientes, se descartaron 7 que no alcanzaban la puntuación para probable o definitiva de HAI, según el protocolo del IAIHG. Se diagnosticaron 81 pacientes con HAI, con edad media de $48,4 \pm 15,7$ (rango 15-84) años, de los cuales $76(93,8 \%)$ eran mujeres de $48,7 \pm 15,9$ años y $5(6,2 \%)$ varones de $43 \pm 12,1$ años, no existiendo diferencias de edad con relación al sexo $(F=0,6 ; p=0,43)$. En la figura 1 se detalla la distribución de las edades de los pacientes al momento del diagnóstico.

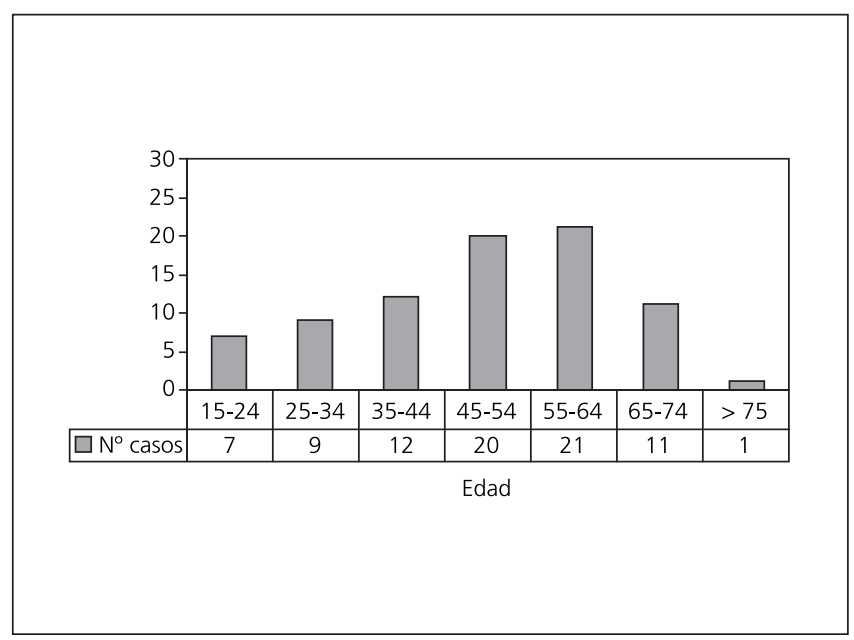

Fig. 1. Distribución de 81 casos de hepatitis autoinmune según la edad de los pacientes al momento del diagnóstico.

Las características de la población del estudio se decriben en la tabla II.

El diagnóstico, aplicando los criterios del IAIHG, fue definitivo en 49 casos y probable en 32. Los diagnósticos pretratamiento se mantuvieron después del tratamiento en la mayoría de los casos ( 78 de 81). De los 3 restantes, 2 pasaron de definitivo a probable y 1 de probable a definitivo. Según la presencia de tipo de autoanticuerpos, 73 pacientes $(90,1 \%)$ fueron clasificados como HAI tipo $1 \mathrm{y}$ $5(6,2 \%)$ como tipo 2 . En los restantes 3 pacientes, el perfil de autoanticuerpos estableció el diagnóstico del tipo de hepatitis autoinmune como indeterminada.

La forma de presentación, en el total de HAI, fue en 35 (43\%) asintomática, en 22 (27\%) como hepatitis aguda, de los que un caso desarrolló insuficiencia hepática aguda grave, y en 24 (30\%) con síntomas de enfermedad hepática crónica, de los que 4 lo fueron como descompensa-
Tabla II. Características de 81 casos de hepatitis autoinmune

\begin{tabular}{|c|c|}
\hline \multicolumn{2}{|l|}{ Edad media (en años) } \\
\hline Hombre (5) & $43 \pm 12,1$ \\
\hline Mujer (76) & $48,7 \pm 12,1$ \\
\hline \multicolumn{2}{|l|}{ Según tipo } \\
\hline Tipo 1 (73) & $50,5 \pm 14,5$ \\
\hline Tipo 2 (5) & $29,2 \pm 12,5$ \\
\hline Indeterminada (3) & $36,7 \pm 23$ \\
\hline \multicolumn{2}{|l|}{ Formas clínicas de presentación } \\
\hline Asintomáticas & $35(43 \%)$ \\
\hline Hepatitis aguda & $22(27 \%)$ \\
\hline Enfermedad hepática crónica & $24(30 \%)$ \\
\hline Enfermedad autoinmune asociada & $20(24,7 \%)$ \\
\hline Hipotiroidismo & 10 \\
\hline Sjögren & 2 \\
\hline Art. reumatoidea & 2 \\
\hline Vitíligo & 2 \\
\hline Otras & 4 \\
\hline \multirow{2}{*}{\multicolumn{2}{|c|}{$\begin{array}{l}\text { Estudio histológico (74 casos) } \\
\text { Grado de actividad }\end{array}$}} \\
\hline & \\
\hline AO & $6(8,1 \%)$ \\
\hline A 1 & $16(21,6 \%)$ \\
\hline A 2 & $35(47,3 \%)$ \\
\hline A 3 & $17(23 \%)$ \\
\hline \multicolumn{2}{|l|}{ Estadio de fibrosis } \\
\hline FO & $9(12,2 \%)$ \\
\hline F1 & $18(24,3 \%)$ \\
\hline $\mathrm{F} 2$ & $9(12,2 \%)$ \\
\hline F3 & $14(18,9 \%)$ \\
\hline F4 & $24(32,4 \%)$ \\
\hline \multicolumn{2}{|l|}{ Diagnóstico (81 casos) } \\
\hline Hepatitis crónica sin cirrosis & $53(65,4 \%)$ \\
\hline Cirrosis hepática & $28(34,6 \%)$ \\
\hline \multicolumn{2}{|c|}{ Relación cirrosis hepática y edad de comienzo de enfermedad } \\
\hline$<60$ años & $17 / 63(26,9 \%)$ \\
\hline$>60$ años & $11 / 18(61,1 \%)$ \\
\hline \multicolumn{2}{|c|}{ Relación cirrosis hepática y forma clínica de presentación } \\
\hline Asintomáticos & $9 / 35(25,7 \%)$ \\
\hline Hepatitis agudas & $7 / 22(31,8 \%)$ \\
\hline Enfermedad hepática crónica & $12 / 24(50 \%)$ \\
\hline \multicolumn{2}{|l|}{ Tratamientos inmunosupresores } \\
\hline Indicado tratamiento inducción & $57 / 81(70,4 \%)$ \\
\hline Respuesta inicial & $54 / 57(94,7 \%)$ \\
\hline Tiempo medio obtención respuesta inicial & $8 \pm 11$ semanas \\
\hline Recaidas & $17 / 54(31,5 \%)$ \\
\hline Durante tratamiento & $3 / 31(9,7 \%)$ \\
\hline Tras retirada de tratamiento & $14 / 23(60,9 \%)$ \\
\hline
\end{tabular}

ción (ascitis o hemorragia digestiva alta) de cirrosis hepática (Tabla II).

En el momento del diagnóstico 72 (89\%) pacientes presentaban elevación de ALT. Las gammaglobulinas se encontraron con valores superiores a 2 veces el valor normal en la mayoría $(62 \%)$ de los casos, entre 1,5 y 2 veces en el $17 \%$ y entre $1-1,5$ veces en el $21 \%$ de los pacientes.

El estudio histológico hepático fue posible realizarlo en 74 pacientes. En otros 4 pacientes el diagnóstico de cirrosis fue realizado por datos clínicos, bioquímicos y radiológicos. Una paciente fue diagnosticada durante el primer trimestre de embarazo. Los otros 2 pacientes rehusaron la biopsia hepática, excluyéndose el diagnósti- 
co de cirrosis por las manifestaciones clínicas, test analíticos, prueba de función hepática y radiología.

De los 74 pacientes a los que se les practicó estudio histológico con biopsia hepática, en 71 existía infiltrado linfoplasmocitario, en 52 lesiones de la limitante y en 15 se encontraron lesiones con distribución en rosetas. Atendiendo a la gravedad, 24 se encontraban en fase de cirrosis, 23 con estadio de fibrosis 2 ó 3, mientras que 27 se diagnosticaron con lesiones leves (estadios de fibrosis 01). En la tabla II se detallan los resultados histológicos.

Los pacientes con cirrosis presentaron con más frecuencia edades superiores a 60 años $(61 \%)$ que los pacientes sin cirrosis (27\%) (Chi cuadrado $=7,2 ; \mathrm{p}=0,01$ ), mientras que no se encontraron diferencias entre los pacientes con o sin cirrosis según la forma de comienzo de la enfermedad.

Al comparar la HAI según los tipos 1 ó 2, los pacientes con tipo 2 eran más jóvenes $(29,2 \pm 11,2$ versus $50,5 \pm$ 14,2 años; $F=12,5 ; \mathrm{p}=0,007$ ) y con predominio de la forma de presentación de hepatitis agudas $(66,7$ versus $26,6 \%$, Chi cuadrado $=3,86, \mathrm{p}=0,04$ ).

Existían enfermedades autoinmunes asociadas en 20 $(24,7 \%)$ pacientes, de las cuales la más frecuente fue el hipotiroidismo (Tabla II). No existieron diferencias en la presentación de hipotiroidismo entre los pacientes de edad inferior $(14 / 62,22,5 \%)$ o superior $(6 / 18,33,3 \%)$ de 60 años (Chi cuadrado $=0,8, \mathrm{p}=0,36$ ).

El tratamiento inmunosupresor se indicó en $57(70,4 \%)$ de los pacientes, principalmente con prednisona sola o asociada con azatioprina (39 y 18 respectivamente), obteniéndose remisión completa o parcial en el 87,7\% de los casos. En 7 de los pacientes se administró también ácido ursodesoxicólico. El tiempo medio de obtención de la respuesta fue de $8 \pm 11$ semanas. La recaída tras la retirada de la inmunosupresión ocurrió en el $61 \%$ de los pacientes (Tabla II), reinstaurándose en ellos el tratamiento previo. No se han encontrado diferencias significativas de la respuesta al tratamiento según la forma clínica de presentación $(\mathrm{p}=$ $0,46)$ ni el tipo de HAI $(p=0,86)$.

\section{DISCUSIÓN}

Se realiza un estudio observacional para conocer las características clínicas, subtipos, enfermedades autoinmunes asociadas y respuesta a la terapéutica de la HAI en el adulto de la provincia de Valencia, puesto que se ha indicado que existen diferencias geográficas en estos factores (2-4). La población estudiada consistía en todos los pacientes atendidos en los servicios de digestivo de ocho hospitales participantes en el estudio durante el año 2003, tanto los diagnosticados ese año como los que venían siendo controlados periódicamente y habían sido diagnosticados 10 años antes.

En contraste con lo encontrado en otras regiones geográficas, en donde la forma de presentación como hepatitis aguda (1) o cirrosis (4) era más frecuente, en nuestra zona la forma más frecuente es la presentación asintomática.

En la mayoría de los casos estos pacientes, sin manifestaciones clínicas de enfermedad hepática, fueron remitidos para estudio por elevación de transaminasas. Sin embargo no son infrecuentes las formas con presentación sintomática, bien como hepatitis aguda o como descompensación de cirrosis. En concordancia con el estudio de Kogan y cols. (12), la severidad de la lesión histológica era similar entre las formas de presentación sintomáticas y asintomáticas. Tampoco se encontró relación entre la cirrosis y la presentación sintomática o asintomática. En nuestro estudio, como en otros $(13,14)$, la cirrosis era significativamente más frecuente entre los pacientes de más de 60 años de edad. En el estudio de Granito y cols. (13), las alteraciones tiroides autoinmunes se presentaron más frecuentemente entre los pacientes de más de 65 años, mientras que este hecho no ha sido observado en nuestro estudio.

Un hecho común entre las diferentes regiones geográficas es la mayor frecuencia del tipo 1 . Al igual que refieren otros autores $(5,15)$, nosotros también encontramos que las HAI tipo 2 se presentan con más frecuencia en edades más jóvenes y con forma de presentación de hepatitis aguda.

Hemos aplicado las normas terapéuticas recomendadas por la mayoría de los autores. La terapéutica inmunosupresora no se indicó en 24 pacientes con formas asintomáticas de presentación, mínima actividad inflamatoria en la biopsia hepática y/o valores normales o mínimamente elevados de transaminasas. En una cohorte de 125 pacientes con HAI, la mitad de los pacientes con formas asintomáticas no recibió tratamiento inmunosupresor y su supervivencia no fue diferente (16). Los autores de este estudio concluyeron que los pacientes con presentación asintomática tenían un buen pronóstico y podían no requerir terapéutica inmunosupresora. En nuestra serie la respuesta a la terapéutica inmunosupresora fue buena, con remisión completa o parcial en el 87,7\% de los casos. Sin embargo, tras cesar el tratamiento, la enfermedad se reactivó en el $61 \%$ de los pacientes, reinstaurándose en ellos el tratamiento previo. A este respecto el tiempo de mantenimiento de la terapéutica debe ser evaluado (1719), puesto que bajas dosis de inmunnosupresores pueden ser mantenidas durante años con control de los efectos secundarios (20). Hay que tener también en cuenta que se ha observado una mejoría del pronóstico entre los pacientes que mantienen transaminasas normales durante el tratamiento inmunosupresor (21). Un tratamiento prolongado de la terapéutica inmunosupresora debería ser evaluado en futuros estudios ante la presencia de factores asociados a la recaída, como valores de transaminasas superiores a los normales durante la remisión y elevada actividad de células linfoplasmocitarias inflamatorias tanto en el espacio portal como en el parénquima hepático en la biopsia hepática (6). Además de los aspectos terapéuticos, otro aspecto a considerar en un futuro próximo, tal como se- 
ñalan Cantarero y cols. (22), es la asociación de la HAI con otro tipo de enfermedades, como enfermedad celiaca, que presentan alteraciones de genes similares.

En conclusión, en el área geográfica de la provincia de Valencia, las formas de HAI del adulto son diagnosticadas más frecuentemente en mujeres y en formas asintomáticas. La mayoría de los casos es de tipo 1 y en un $25 \%$ de los casos coexiste otra enfermedad autoinmune. Al momento del diagnóstico, una tercera parte de los pacientes se encuentra en fase de cirrosis, sobre todo entre los que tienen más de 60 años. Los tipo 2 de HAI se presentan con mayor frecuencia en edades más juveniles y como forma de hepatitis aguda. La terapéutica inmunosupresora es efectiva en la mayoría de los pacientes, aunque la recaída tras la retirada de la medicación es un hecho frecuente.

\section{AGRADECIMIENTOS}

Los autores quieren agradecer a la Dra. Marta Pulido la asistencia para la redacción del manuscrito.

\section{BIBLIOGRAFÍA}

1. Ferrari R, Pappas G, Agostinelli D, Muratori P, Muratori L, Lenzi M. Type I autoimmune hepatitis: Patterns of clinical presentation and differential diagnosis of the 'acute' type. QJM 2004; 97: 407-12.

2. Nishioka M, Morshed SA, McFarlane IG. Geographical variation in the frequency and characteristics of autoimmune liver diseases. In: Krawitt EL, Wiesner RH, Nishioka M, editors. Autoimmune liver diseases. Amsterdam: Elsevier; 1998. p. 413-24.

3. Czaja AJ, Souto EO, Bittencourt PL, Cancado EL. Clinical distinctions and pathogenic implications of type 1 autoimmune hepatitis in Brazil and the United States. J Hepatol 2002; 37: 302-8.

4. Choudhuri G, Somani SK, Baba C, Alexander G. Autoimmune hepatitis in India: Profile of an uncommon disease. BMC Gastroenterol 2005; 5: 27.

5. Homberg JC, Abuaf N, Bernard O, Islam S, Álvarez F, Khalil SH, et al. Chronic active hepatitis associated with antiliver/kidney microsome antibody type 1: A second type of "autoimmune" hepatitis. Hepa- tology 1987; 7: 1333-9.

6. Verma S, Gunuwan B, Mendler M, Govindrajan S, Redeker A. Factors predicting relapse and poor outcome in type 1 autoimmune hepatitis: Role of cirrhosis development, patterns of transaminases during remission and plasma cell activity in the liver biopsy. Am J Gastroenterol 2004; 99: 1510-6.

7. Johnson PJ, McFarlane IG. Meeting report: International Autoimmune Hepatitis Group. Hepatology 1993; 18: 998-1005.

8. Álvarez F, Berg PA, Bianchi FB, Bianchi L, Burroughs AK, Cancado EL, et al. International Autoimmune Hepatitis Group Report: Review of criteria for diagnosis of autoimmune hepatitis. J Hepatol 1999; 31 : 929-38.

9. McFarlane IG. Definition and classification of autoimmune hepatitis Semin Liver Dis 2002; 22: 317-24.

10. Czaja AJ, Freese DK. AASLD Practice Guidelines. Diagnosis and treatment of autoimmune hepatitis. Hepatology 2002; 36: 479-97.

11. Bedossa P, Poynard T, for the METAVIR Cooperative Study Group. An algorithm for the grading of activity in chronic hepatitis C. Hepatology 1996; 24: 289-93.

12. Kogan J, Safadi R, Ashur Y, Shouval D, Ilan Y. Prognosis of symptomatic versus asymptomatic autimmune hepatitis: A study of 68 patients. J Clin Gastroenterol 2002; 35: 75-81.

13. Granito A, Muratori L, Pappas G, Muratori P, Ferri S, Cassani F, et al. Clinical features of type 1 autoimmune hepatitis in enderly Italian patients. Aliment Pharmacol Ther 2005; 21: 1273-7.

14. Czaja AJ, Carpenter HA. Distinctive clinical phenotype and treatment outcome of type 1 autoimmune hepatitis in the elderly. Hepatology 2006; 43: 532-8

15. García-Romero R, Martín J, Bernal C, Pinillos S, Varea V. Autoimmune hepatitis in pediatric patients. Rev Esp Enferm Dig 2007; 99: 255-8.

16. Feld JJ, Dinh H, Arenovich T, Marcus VA, Wanless IR, Heathcote EJ. Autoimmune hepatitis: Effect of symptoms and cirrhosis on natural history and outcome. Hepatology 2005; 42: 53-62.

17. Krawitt EL. Medical progress: Autoimmune hepatitis. N Engl J Med 2006; 354: 54-66.

18. Manns MP, Vogel A. Autoimmune hepatitis from mechanisms to therapy. Hepatology 2006; 42: S132-44.

19. Montano-Loza AJ, Carpenter HA, Czaja AJ. Improving the end of corticosteroid therapy in type 1 autoimmune hepatitis to reduce the frequency of relapse. A J Gastroenterol 2007; 102: 1005-12.

20. Seela S, Sheela H, Boyer JL. Autoimmune Hepatitis type 1: Safety and efficacy of prolonged medical therapy. Liver Int 2005; 25: 734-9.

21. Miyake Y, Iwasaki Y, Terado R, Takagi S, Okamaoto R, Ikeda H, et al. Persistent normalization $\mathrm{f}$ serum alanine aminotransferase levels improves the prognosis of type 1 autoimmune hepatitis. J Hepatol 2005; 43: 951-7.

22. Cantarero MD, Gómez J, Menchén L, Pajares JA, LoIacono Q. Liver damage and celiac disease. Rev Esp Enferm Dig 2007; 99: 648-52. 